\title{
Wearable Audio-Feedback System for Gait Rehabilitation in Subjects With Parkinson's Disease
}

Filippo Casamassima DEI - University of Bologna

Via Risorgimento 2

Bologna, Italy

filippo.casamassima@unibo.it
Laura Rocchi

DEI - University of Bologna

Via Risorgimento 2

Bologna, Italy

I.rocchi@unibo.it

\section{Alberto Ferrari}

DEI - University of Bologna

Via Risorgimento 2

Bologna, Italy

alberto.ferrari@unibo.it

Bojan Milosevic

DEI - University of Bologna

Via Risorgimento 2

Bologna, Italy

bojan.milosevic@unibo.it
Elisabetta Farella

DEI - University of Bologna

Via Risorgimento 2

Bologna, Italy

elisabetta.farella@unibo.it

Permission to make digital or hard copies of part or all of this work for personal or classroom use is granted without fee provided that copies are not this notice and the full citation on the first page. Copyrights for third-part this notice and the full citation on the first page. Copyrights for third-party owner/author(s). Copyright is held by the author/owner(s).

UbiComp'13 Adjunct, September 8-12, 2013, Zurich, Switzerland. ACM 978-1-4503-2215-7/13/09.

http://dx.doi.org/10.1145/2494091.2494178

\begin{abstract}
Parkinson's disease (PD) is a degenerative neurological disorder, associated with movement impairments. Recent studies have shown that auxiliary cueing in the form of video, audio, or haptic feedback can improve the gait performance in PD patients. We have developed a new platform to support gait rehabilitation in PD patients based on a wearable system able to produce real-time feedback to the user in a comfortable and effective way. Using a set of custom wearable inertial sensors, with advanced on-board processing capabilities, our application employs a smartphone to analyze in real time the patients gait and to return an appropriate real time audio bio-feedback (ABF) message to the user to correct and improve gait performance. The main advantages of the system are mobility and unobtrusiveness: it can be comfortably worn and carried by the patient with no range restrictions, giving the possibility to monitor and rehabilitate the patient in real-life scenarios, both indoors and outdoors.
\end{abstract}

\section{Author Keywords}

Audio Feedback, Parkinson, Inertial Measurement Unit

\section{ACM Classification Keywords}

J.3 [Life and Medical Sciences]: Health.; H.5.2 [User Interfaces]: Auditory (non-speech) feedback 


\section{Introduction}

Parkinsons disease is an idiopathic condition, often characterized by gait impairments, arising from progressive degeneration of the central nervous system. Some research has shown that external cues in the form of video, audio or haptic feedback can help in movement planning and execution. However, rehabilitation is rarely performed at home autonomously by the patient [3]. The contribution of this work is to present a system for gait rehabilitation for use in a daily life setting, developed in the context of the EU-funded project $C u P i D^{1}$. The goal of the system is to provide real-time audio feedback to the patient, by means of vocal messages. It employs a fully portable and wearable architecture, i.e. a wireless body sensor network, based on inertial measurement units, connected with a smartphone used as a portable processing platform. The therapeutic hypothesis is centered on the concept of motor learning and is based on intensive repetitions of a motor task. The messages are returned to the user by the automatic and stand-alone systems, and they are inspired by the type of vocal instructions given in traditional rehabilitation contexts by clinical operators.

The pervasive use of technology brings many potential advantages to the ambulatory rehabilitation of patients. For example, it is impossible to quantitatively evaluate the patient's improvements; monitor and assist subjects outside the clinic and support them during autonomous exercises. In [3], for example, authors were able to design a shoe capable of reading pressure values from each foot and give back a vibratory feedback, with the aim to improve gait of PD patients. Promising preliminary results were shown, but the system is complex to use even for expert clinicians and requires high power consumption.

\footnotetext{
${ }^{1}$ Closed loop system for personalized and at home rehabilitation of Parkinsons disease - www. cupid-project.eu
}

Other works combine the use of auditory, video and tactile feedback [1, 2]. In [2], for example, a mobile phone is used as to return the feedback to the user, while pressure sensors mounted in the shoes are used as inputs.

Limitations of this promising approach include a poor clinical validation, its use mainly in a supervised condition and the impossibility to extend the approach to motor tasks different than gait.

The system we propose in this work uses wearable inertial measurement units (IMUs) and can be constituted by a variable number of IMUs to monitor different parts of the body. Moreover, each sensing node is equipped with a high performance microcontroller (MCU), capable to process the sensor data with the advantage to reduce both the network traffic and the phone's workload.

\section{System Architecture}

The ABF system, designed and developed in the context of the CuPiD project, is essentially composed by three elements: the inertial sensor nodes, an Android mobile phone and the ABF application. The hardware components will be presented in this section, while the applications and the related algorithms will be introduced in the next one.

\section{Inertial sensing nodes}

The sensor nodes have been designed keeping in mind their main target use, i.e. the analysis and monitoring of human movements, with a specific emphasis on gait. Each node is equipped with an STM32F103 MCU based on an ARM Cortex M3 core running at $72 \mathrm{MHz}$. In addition, they embed a combination of MEMS sensors by ST (gyroscope, accelerometer and magnetometer), which can take advantage of sleep states to reduce power consumption. The device is provided with a Bluetooth module, which also utilizes the standard Bluetooth low power modes 
Headphones

for auditory feedback

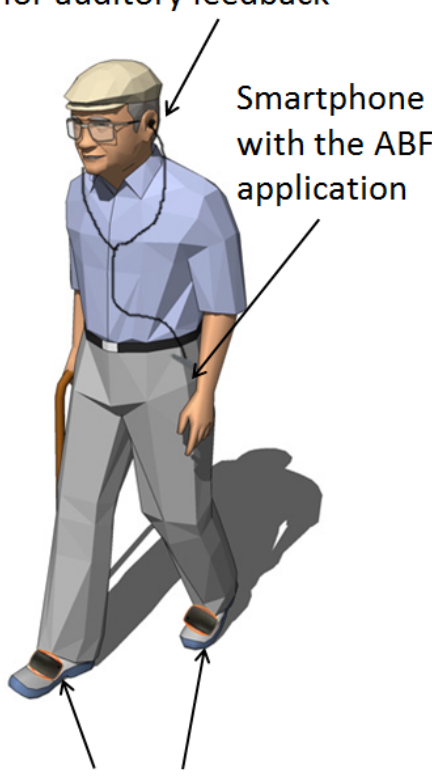

Inertial sensors

Figure 1: ABF system weared by a PD patient (sniff, park and hold) and provides the ability to build a network of up to 7 nodes and to communicate with a vast range of devices through the Serial Port Protocol (SPP). The hardware configuration is completed by a 1 Gb NAND ash memory employed for local data storage.

The hardware and firmware have been designed to allow maximum flexibility and to use them for different scenarios (i.e. posture recognition, trunk movements). It is indeed possible to use the nodes for data streaming, data logging and on-board processing, thanks to an embedded version of the Kalman Filter implemented to process the sensor data and estimate the device's orientation.

\section{Mobile phone}

An Android mobile phone has been chosen to manage the network, send commands and receive data from the sensor nodes. Requirements in this case are not strict: a phone with a Bluetooth connection capable to run android 2.3 or higher is enough. The application developed is capable to receive raw data or the pre-computed orientation from the sensor nodes. It returns an audio feedback to the patient, according to the parameters selected by the clinicians during a training phase.

\section{Real-time Audio Feedback Application}

Clinical goal

Spatio-temporal gait parameters, such as cadence, gait speed, etc., can provide the information needed to monitor the performance of a subject's walk and an accurate measure of the overall efficacy of the locomotor function. The use of IMUs allows to detect and record all the spectrum of gait parameters needed for an accurate analysis. To provide a useful real-time feedback to improve gait performance, the gait performance of PD patients has to be computed and fed back in an efficient way to the patients, using auditory signals. Following clinical practice and feasibility criteria, the gait parameters selected as most relevant for PD patients are: cadence, step length, trunk posture, gait speed, gait asymmetry. The computation of all these parameters in real time was implemented into the ABF Application. A new online automatic algorithm was developed to estimate the gait events. The algorithm determines foot initial contact and foot off by processing the angular velocity along the medio-lateral axis of the IMUs rigidly attached to the patient's feet. Once these events are detected, it is possible to determine the previously described spatio-temporal gait parameters and evaluate the nature of the walk performed by the patient.

Overall, the functional goal of the system entails the improvement of gait's rhythm and amplitude, the decrease of the asymmetry and the correction of a wrong posture assumption.

\section{Usage scenario}

The ABF system will be delivered to patients to be used at home to support the gait training over a period of 6 weeks. The typical scenario of use of the system entails the patient walking freely outdoors, in a park for example, over single or multiple periods of 30 minutes each. During a training session, the ABF system measures the selected gait parameters and compares them with respect to reference values over windows of some steps (e.g. every 5 steps). Positive vocal information is returned in case the patient is walking close to his/her specific reference values, support messages are given every time the patient is below or above the target zone. Moreover, the system is able to monitor any abnormal forward inclination of the trunk, being this a strong disabling symptom in some PD patients. Practically, the reference values for each patient will be set before the beginning of the training campaign, by means of the execution of a gait trial supervised by 


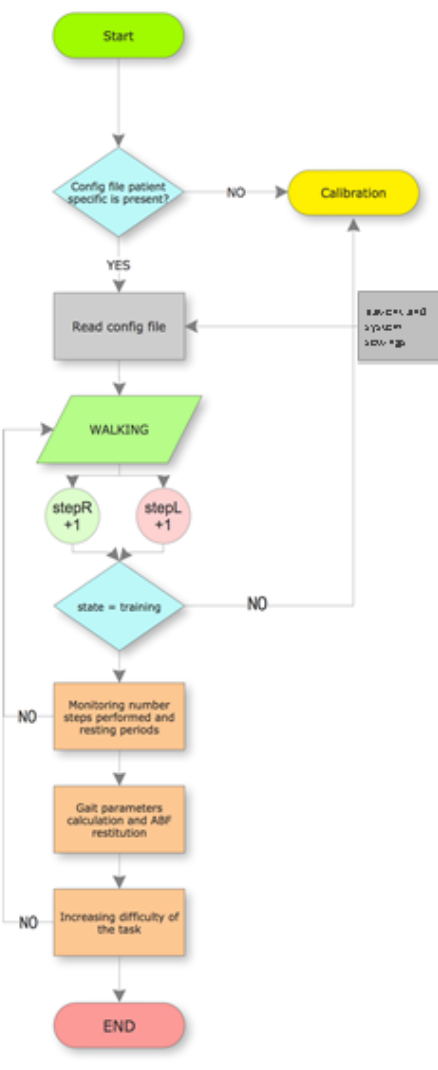

Figure 2: Block diagram of the $\mathrm{ABF}$ application. clinical staff. The concept is to provide patients with tools to re-learn effective patterns and posture, and to develop new strategies to overcome the disability and perform every day at their best.

\section{ABF application and algorithms}

To make the system usable during the daily life of PD patients, it is necessary to make it available to the end-user through an extremely easy-to-use system and user-friendly application. In order to achieve these goals, the body area network was limited to the use of 3 IMUs, 2 placed on top of the shoes and one on the lower trunk. The ABF application was developed using the Matlab GUI Builder (MathWorks, USA), then the software architecture and algorithms were ported in Java and included as a library in Android, to realize a smartphone stand-alone application. The software developed resulted in an event-driven finite state machine with 4 available states: disconnected, connected, calibrated and training. Once the system is calibrated, it allows the patients to execute the ABF training by simply pressing a single button at the beginning of the session avoiding to handle the IMUs connections or other ABF settings (see Fig. 2). In particular, the software architecture is formed of 6 components: 1) clinical database; 2) connecting routine: automatic Bluetooth connection of IMUs to the smartphone; 3) calibrating routine: special walking acquisition aimed to set the patient's reference gait parameters; 4) loading of system and patient specific settings, concerning spatio-temporal parameters selected and feedback restitution 5) recording of the raw IMUs data and real-time gait event recognition; 6) ABF running routine: reward or support through vocal feedback; 7) generation of log files and clinical summary of the recorded session. Noteworthy, the application is able to automatically increase the difficulty of the task, once the patient is able to remain constantly in the target zone during a training session.

\section{Conclusions}

In this work we presented a system for gait rehabilitation for use in a daily life setting. By embracing a fully portable and wearable solution, the proposed system aims to support patient's gait rehabilitation and provides real-time audio feedback by means of vocal messages. The system is now being used for pilot trials with PD patients, whose feedback and comments will be employed to adjust the application for a more extensive clinical usage.

\section{Acknowledgements}

The research leading to these results has received funding from the European Union - Seventh Framework Programme (FP7/2007-2013) under the grant agreement n.288516 (CuPiD project).

\section{References}

[1] Baram, Y., and Lenger, R. Gait improvement in patients with cerebral palsy by visual and auditory feedback. In Intl. Conf. on Virtual Rehabilitation (2009), 146-149.

[2] Redd, C., and Bamberg, S. A wireless sensory feedback device for real-time gait feedback and training. IEEE/ASME Transactions on Mechatronics 17, 3 (2012), 425-433.

[3] Winfree, K., Pretzer-Aboff, I., Hilgart, D., Aggarwal, R., Behari, M., and Agrawal, S. An untethered shoe with vibratory feedback for improving gait of parkinson's patients: The pdshoe. In Proc. Engineering in Medicine and Biology Society (EMBC) (2012), 1202-1205. 